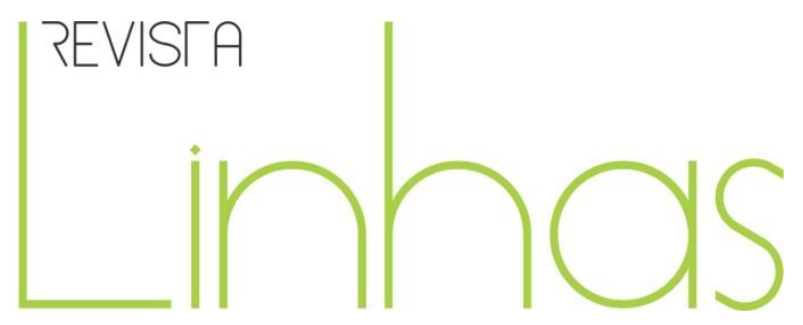

\title{
"O árduo caminho": Memórias de um inspetor escolar italiano (Berengario Galileo Amorosa, 1865-1937)
}

\section{Resumo}

Este artigo propõe um estudo de caso das memórias do serviço escolar publicadas em 1912 pelo inspetor escolar italiano Berengario Galileo Amorosa (intelectual, historiador e erudito), visando focar a dupla oportunidade que fontes similares oferecem à pesquisa. Por um lado, as páginas de Amorosa remetem a uma reflexão metodológica em torno da "versatilidade" dessa fonte historiográfica, pairando entre testemunho e narrativa, marca pessoal e escrita pública de difícil definição, como sugerido por Vinao Frago. Por outro, incitam uma reconstrução mais detalhada da "história da inspetoria" na área italiana, ainda em grande parte a ser escrito. Interessa, aqui, o gênero literário representado pelas memórias autobiográficas de inspetores, do qual o texto de Amorosa oferece um modelo significativo. A meio caminho entre registro e memória pessoal, o conhecimento sobre o mundo escolar é restituído pelas memórias e histórias de um observador "especial”: emerge uma representação da escola entre o fim do século XIX e início do século XX que, além de evocar "as angústias, misérias e tristezas da classe magistral", restitui informações sobre o cotidiano escolar por meio da "representação do ambiente complexo". As histórias são confiadas a uma chave evocativa e narrativa pessoal que não podem naturalmente prescindir do âmbito da experiência coletiva que ocorre em segundo plano. Neste sentido, adquire importância o trabalho de Amorosa, que reflete "fielmente catorze anos de vida dedicada ao ensino fundamental, entre povos e cidades distintos", no decorrer do "árduo caminho" nas primeiras décadas do século XX. Suas memórias, em última análise, contribuem enquanto gênero textual para a compreensão aproximada dos principais processos de mudança educacional que atravessaram a vida escolar italiana entre os séculos XIX e XX.

Palavras-chave: Inspetor Escolar. História da Inspetoria. Vida Escolar Italiana. Séculos XIX e XX.

\section{Para citar este artigo:}

D’ALESSIO, Michela. "O árduo caminho": Memórias de um inspetor escolar italiano (Berengario Galileo Amorosa, 1865-1937). Revista Linhas. Florianópolis, v. 18, n. 36, p. 200-220, jan./abr. 2017. Título original: "Il duro calle". Le memorie di un ispettore scolastico italiano (Berengario Galileo Amorosa - 1865/1937). Traduzido e revisado por Andressa Picosque.

DOI: $10.5965 / 1984723818362017200$

http://dx.doi.org/10.5965/1984723818362017200

\author{
Michela D'Alessio \\ Universidade de Estudos de \\ Basilicata - Itália \\ michelina.dalessio@unibas.it
}




\title{
"The hard road": The memories of an Italian school inspector (Berengario Galileo Amorosa - 1865/1937)
}

\begin{abstract}
This paper proposes a case study constituted by school memories published in 1912 by the Italian inspector Berengario Galileo Amorosa (intellectual, historian and scholar), with the objective of calling attention to the double opportunity that a similar kind of source offers to the research. On the one hand, the pages of Amorosa send us back to methodological reflections on the "versatility" of this historiographical source, poised between testimony and narrative, personal mark and public writing, of difficult definition, as suggested by Vinao Frago. On the other hand, they impel towards a more detailed reconstruction of the "history of the inspection function" in the Italian area, still largely to be written. At this point, the interest is directed to the literary text genre represented by the autobiographical memories of the inspectors, from which the text of Amorosa offers a significant example. Halfway between report and personal remembrance, the knowledge about the school world is restored from the memories and stories of a "special" observer: what emerges is a depiction of the school in the late nineteenth and the beginning of the twentieth century, which, apart from recalling "the distress, the miseries and sorrows of the class of teachers", gives us information about the everyday school life, through representation of this manifold environment. The stories are entrusted to a recollection character and personal narrative that naturally cannot renounce the framework of collective experience that is placed in the background. In this sense, the work of Amorosa gains importance, which "faithfully reflects fourteen years of life dedicated to the elementary school in different countries and among different peoples", along the "hard road" in the early decades of the twentieth century. Ultimately, his memoirs, as text genre, contribute in a very close way for the understanding of the major processes of educational change experienced by the Italian school life in the nineteenth and twentieth centuries.
\end{abstract}

Keywords: Scholar Inspector. History of the Inspection function. Italian School Life. Nineteenth and Twentieth Centuries. 
Tu proverai sì come sa di sale Lo pane altrui, e com'è duro calle Lo scendere e il salir per l'altrui scale

(Dante, Paradiso, XVII)

\section{Introdução: um estudo de caso italiano sobre inspeção escolar}

Este artigo propõe o estudo de caso das memórias do serviço escolar publicadas em 1912 pelo inspetor escolar italiano Berengario Galileo Amorosa', com o intuito de focar as principais oportunidades que fontes similares garantem à pesquisa. $\mathrm{O}$ olhar focado nos episódios biográficos e na produção de estilo "escolar" do respeitável representante de Molise irá convergir para um exame específico das suas memórias autobiográficas, qualificadas em sua natureza exemplar, tanto pelos aspectos de reflexão metodológica e historiográfica que incentiva quanto pelas hipóteses de reconstrução de um esboço da inspetoria italiana entre os séculos XIX e XX.

\section{A fonte de estudo e as oportunidades cognitivas: os ego-documentos e a inspetoria italiana}

Por um lado, as páginas de Amorosa levam a uma reflexão metodológica em torno da "versatilidade" dessa fonte historiográfica, a meio caminho entre testemunho e narrativa, cuja discussão será retomada em breve. Uma escrita que se apresenta como autorreferencial - no sentido de que o sujeito fala de si mesmo - está ao mesmo tempo focada no relato das relações estabelecidas pelo inspetor com os lugares e os protagonistas do mundo escolar que relata, naturalmente segundo seu olhar e, portanto, um modelo pessoal de representação. A esse respeito remete-se, em particular, à dificuldade de definir tais fontes ${ }^{2}$, persuasivamente exposta por Vinao Frago ${ }^{3}$.

\footnotetext{
${ }^{1}$ B.G. Amorosa, II "duro calle". Memorie d'un ispettore scolastico, Milano Roma - Napoli, Società editrice Dante Alighieri di Albrighi \& C., 1912. Um resumo da pesquisa realizada em torno da figura do inspetor Amorosa foi inicialmente apresentado na obra M. D'Alessio, "Il duro calle". Memorie di un ispettore scolastico (Berengario Galileo Amorosa, 1865-1937), in C. Yanes, J. Meda (Ed.), International Symposium, School memories. New trends in Historical research into Education Heuristic Perspectives and Methodological issues (Sevilha, 22-23 de setembro de 2015). Abstracts, p. 22.

${ }^{2}$ Sobre o tema dos "ego-documentos" encontrados nos escritos de professores - e aqui de inspetores recomenda-se as considerações expostas especificamente sobre registros escolares em M. D'Alessio, Life at
} 
Por outro lado, incitam a reconstrução da "história da inspetoria" italiana4. Um primeiro passo nesta direção se origina nos anos 1960 por Giacomo Cives com a recuperação de alguns documentos e testemunhos de inspetores, visitantes e educadores ${ }^{5}$. 0 estudioso atribuiu os elementos críticos da inspeção italiana tradicional à confusão das suas tarefas e ao seu campo de ação indistinto, que oscila entre a função técnico-educacional e aquela mais propriamente administrativa. Visitantes, observadores dos acontecimentos de muitas escolas, os inspetores têm contribuído para o conhecimento concreto das diferentes situações em que se faz escola na Itália. Seus relatórios, que a historiografia escolar-educacional não estuda adequadamente, recuperam informações nada secundárias sobre suas "viagens pedagógicas". Produzindo documentos muitas vezes não só burocráticos e administrativos, as visitas de inspeção nos apresentam o conhecimento de fatos e situações precisas e concretas em termos de aplicações práticas das orientações didático-pedagógicas para ajudar professores e do registro de investigações ambientais e sociológicas.

Nessa linha se encaixa o mesmo interesse na experiência da personalidade considerada "mais autenticamente de inspeção em constituição e temperamento", a de Giuseppe Lombardo Radice ${ }^{6}$. O trabalho com o qual me interessei pela reconstrução de um quadro eficaz - dado que proveniente de uma perspectiva contemporânea - da

school: class registers as a new source of studying historical and educational heritage, in A.M. Badanelli Rubio, M. Poveda Sanza, C. Rodriguez Guerrero (Ed.), Pedagogia museistica. Practicas, usos didacticos e investigacion del patrimonio educativo, Atti della VI Jornadas Cientificas del la Sociedad Española para el Estudio del Patrimonio Histórico Educativo SEPHE (Madri, 22-24 de outubro de 2014), Madrid, Universidad Complutense de Madrid, Facultad de Educacion, 2014, pp. 401-409.

${ }^{3} \mathrm{Cf}$. A. Vinao Frago, Relatos y relaciones autobiograficas de profesores y maestros, in A. Escolano Benito, J.M. Hernandez Diaz (Ed.), La memoria y el deseo. Cultura de la escuela y educacion deseada, Valencia, Tirant Lo Blanch, 2002, pp. 135-175.

${ }^{4}$ Sobre as transformações do instituto de inspeção escolar, recomenda-se o trabalho de G. Decollanz, La funzione ispettiva dalla legge Casati ad oggi, Roma, Armando, 1984, a que, como foi observado por muitos, não se seguiu uma reconstrução temática de perfis individuais e características de toda a inspetoria.

${ }^{5} \mathrm{G}$. Cives, Cento anni di vita scolastica in Italia. Ispezioni e inchieste da Gino Capponi a Giuseppe Lombardo Radice, Roma, Armando Editore, 1960 e Id., Cento anni di vita scolastica in Italia. Ispezioni e inchieste dall'idealismo a oggi negli scritti di G. Lombardo Radice, G. Isnardi, G. Giovinazzi, F. Betttini, A. Marcucci, L. Volpicelli, L. Borghi, A. Visalberghi, Ibid., 1967.

${ }^{6}$ A ligação entre os amplos esforços educacionais do pedagogo siciliano e sua função de "inspetor nato" não foi sempre suficientemente enfatizada: de fato, "fosse orientador da Associazione del Mezzogiorno ou diretor geral, visitante não oficial ou professor universitário, sabia descobrir as escolas mais modestas e humildes onde se fazia algo de bom da mesma maneira que estudava aquelas mais estabelecidas e relevantes para divulgar os achados, os frutos, as realizações; sabia apoiar e ajudar cada professor, mesmo na situação mais diferente ou difícil ou incomum". Ibid., p. 30. 
aplicação da reforma do sistema educacional de 1923 nos diferentes distritos escolares da Itália obtém um tipo de fonte para a escola de tal forma renovado, entre documentos que, em particular, Lombardo Radice considerava "significativos para a história da escola italiana do nosso tempo"7 mais precisamente em relação aos "relatórios anuais que os inspetores escolares costumam escrever" ${ }^{8}$. Aprofundar-se "nos modos de 'fazer escola' atrai, de fato, hoje mais do que nunca, o olhar dos historiadores da educação, visto que representa a maneira de penetrar o interior da investigação detalhada dos fatores, dos mecanismos, dos desvios e das diferentes dinâmicas por meio dos quais as disposições ministeriais e projetos pedagógicos puderam transmitir em uma rede feita de tramas mais largas, mas também mais intransponíveis, até chegar às realidades individuais das escolas em todo o território nacional"9.

Estímulos mais recentes foram tirados de Giorgio Chiosso, que se ateve não só ao estudo do papel dos inspetores escolares na supervisão do cumprimento das leis e regulamentos, bem como na divulgação de novos métodos de ensino, mas também à necessidade de uma revisão sistemática sobre os inspetores italianos ${ }^{10}$. De fato, o estudioso lamenta a falta de informações sobre o corpo geral dos inspetores escolares italianos em relação às revisões sistemáticas existentes, por exemplo, no contexto francês, e a necessidade de se considerar um reconhecimento arquivístico mais global. Observações nesse sentido, junto a considerações pontuais sobre as características e orientações do corpo de inspeção nos anos da Direita histórica, têm sido útil e recentemente propostas no trabalho documentado de Alberto Barausse em torno das investigações de Bargoni e Bonghi sobre a questão dos livros didáticos durante os primeiros quarenta anos pós-unificação italiana ${ }^{11}$. Alguns pontos de interesse em torno da

\footnotetext{
7 G. Lombardo Radice, Presentazione in R. Dal Piaz, Esperienze didattiche di un ispettore trentino: relazionestudio sulle scuole della circoscrizione di Trento (1925-1926), Roma, Associazione per il Mezzogiorno, 1928, p. 3.

8 O interesse pelas "experiências didáticas" dos inspetores escolares é fator relevante da dimensão historiográfica a que diz respeito meu trabalho sobre manuais regionais introduzidos após a reforma Gentile do sistema educacional italiano: cf. M. D’Alessio, A scuola fra casa e patria. Dialetto e cultura regionale nei libri di testo durante il fascismo, Coleção Biblioteca do "Centro di documentazione e ricerca sulla storia delle istituzioni scolastiche, del libro scolastico e della letteratura per l'infanzia" da Università degli Studi del Molise, Lecce, Pensa Multimedia, 2013.

${ }^{9}$ Ibid., p. 38.

${ }^{10} \mathrm{G}$. Chiosso, Alfabeti d'Italia. La lotta contro l'ignoranza nell'Italia unita, Torino, SEI, 2011, pp. 199-203.

11 “Os relatórios ajudam, assim, até mesmo a penetrar os mecanismos de uma profissão particularmente onerosa e desafiadora. Na verdade, pouco se sabe da identidade e da realidade daqueles que participaram
} 
imprensa técnica especializada nos anos entre idealismo e fascismo também foram abordados pela obra que lançou luz sobre a experiência da revista profissional La Tecnica Scolastica, dirigida por Alfredo Saraz ${ }^{12}$.

Aqui não interessa predominantemente, no entanto, os relatórios específicos das visitas de inspeção - ainda que não adequadamente estudados pela historiografia educacional-escolar enquanto textos institucionais, sempre pairando entre função burocrática e técnico-didática. Considera, em vez disso, principalmente o gênero literário representado pelas memórias autobiográficas de inspetores, do qual o texto de Amorosa oferece um modelo significativo.

\section{O perfil biográfico e do "homem da escola"}

Para saber mais sobre as características da biografia institucional do intelectual, historiador e erudito dedicado à carreira escolar e ministerial, é útil retomar a mesma Autobiografia do autor presente na obra In memoria del Gr.Uff. Berengario Galileo Amorosa

\footnotetext{
da inspetoria nos anos da direita histórica. Infelizmente, não existem estudos sistemáticos que ofereçam um panorama abrangente das características do corpo de inspeção italiano, componente importante da nova e mais generalizada administração escolar italiana. Identificar alguns desses estudos pode, no entanto, ajudar a entender melhor as diferentes e heterogêneas abordagens dos funcionários periféricos sobre a questão dos livros didáticos": cf. A. Barausse, Nonostante tanto diluvio di libri scolastici. I libri di testo per le scuole elementari e le indagini ministeriali di Bargoni e Bonghi durante gli anni della Destra storica (18691875), Lecce, Pensa Multimedia, 2015, p. 20. Referências úteis em torno da formação do corpo de inspeção na era giolittiana estão presentes já em A. Barausse, I maestri all'università: la Scuola pedagogica di Roma 1904-1923, Perugia, Morlacchi, 2004, passim.

${ }^{12}$ Sobre essa imprensa especializada profissional específica que encontrou na Tecnica Scolastica um "órgão sereno e respeitável de homens da escola" e sob a liderança de seu ativo diretor Alfredo Saraz tornou-se uma "oficina" educacional e administrativa singular durante seus cinco anos de vida, de 1923 a 1927, cf. A. Barausse, M. D’Alessio, “La salvezza della scuola verrà dai tecnici”. La stampa pedagogica in Italia tra idealismo e fascismo: la «Tecnica scolastica» (1923-1927), in J.M.H. Diaz (Ed.), La prensa de los escolares y estudiantes. Su contribucion al patrimonio historico educativo, Salamanca, Ediciones Universidad de Salamanca (Collecion Aquilafuente, 210), 2015, pp. 611-626. A revista demonstra seguir desde sua estreia um programa bem definido, ocupando-se "de didática e de administração escolar para todo tipo de escola e série" e apresentando-se ao mesmo tempo como "órgão de consulta, estudo, incentivo para o melhor do nosso mundo escolar". Os principais colaboradores da comissão editorial vêm do mundo dos diretores e dos inspetores escolares. Algumas figuras desempenharam papéis importantes na administração escolar central ou periférica ou no exterior. Entre eles, professores e diretores ou inspetores, como Giovanni Di Giusto, Libero Forte, Amedeo Cominetti, Giacomo Carenzi, Evaristo Marsili, Michele Angelastri. A estes são adicionados os perfis mais próximos à personalidade e ao neoidealismo de Lombardo Radice, entre os quais se destacam os inspetores escolares Riccardo Dal Piaz, Giovanni Lucaroni, Edoardo Predome, junto a ocasionais nomes de maior prestígio, Vidari, Giovanazzi, Tauro, e outros ainda oriundos do mundo magistral feminino, incluindo Amalia Paluffo. A revista passou de um apoio incondicional da "revolução escolar em marcha" da reforma iniciada por Gentile para uma posição mais crítica nos últimos anos, refletindo no geral a heterogeneidade dos diversos componentes do fascismo escolar e pedagógico italiano. Ibidem.
} 
Provveditore agli studi ${ }^{13}$. Iniciado nos estudos elementares pelo pai, distinto educador da comunidade de Riccia ${ }^{14}$, Berengario frequentou o ginásio em vários internatos, primeiro em Assis, em 1875, e depois de 1879 em Nápoles, onde, no ano seguinte, obteve o diploma. Voltou a estudar no prestigiado internato Mario Pagano, em Campobasso ${ }^{15}$, em 1880, e obtém diploma liceal em Nápoles, em 1884-85, além de licença elementar de ensino superior na Escola Normal partenopeia. Logo depois, ensinou por cinco anos em sua cidade de origem ${ }^{16}$ e em seguida foi enviado a Montenero di Bisaccia até 1891.

De fato, em fevereiro do mesmo ano, Amorosa foi aprovado no concurso para inspetor escolar, com destino a Conegliano, no Vêneto. Começa a trabalhar na comuna trevisana em 27 de março de 1891, permanecendo ali por um ano. É sabido que a unidade política e administrativa alcançada incentivou inúmeras transferências de oficiais e funcionários públicos, principalmente professores, à luz de uma nova sociedade nacional em que se favorecia mais ampla circulação de pessoas e de ideias que no passado. De acordo, portanto, com a mobilidade profissional e didática difundida naquele tempo, o molisano esteve em Pieve di Cadore (de 12 de janeiro de 1892 a 30 de setembro de 1893), Sulmona (de $1^{\circ}$ de outubro de 1893 a 6 de novembro de 1895), Penne, sempre em Abruzzo (de 7 de novembro de 1895 a 10 de novembro de 1897); então na Sardenha, em Sassari (de 11 de novembro de 1897 a 13 de abril de 1898) e por um longo período em Lanciano

\footnotetext{
${ }^{13}$ B.G. Amorosa, Autobiografia, in In memoria del G. Uff. Dottor Berengario Galileo Amorosa Provveditore agli studi, MDCCCLIV-MCMXXXVII, s.n.t. [1938], pp. 11-23. Recentemente, o perfil de Amorosa foi proposto por M. D’Alessio, ad vocem no repertório do DBE Dizionario Biografico dell'Educazione. 1800-2000, dirigido por Giorgio Chiosso e Roberto Sani, Milano, Editrice Bibliografica, 2013, 2 v., v. I, nota $n^{\circ} 75$, pp. 45-46. Uma profícua revisão das obras sobre a personalidade e o papel de Amorosa se origina no volume de G. Palmieri, A. Santoriello (Ed.), Berengario Galileo Amorosa. Atti del Convegno, Riccia, 18 luglio 1987, Riccia, Associazione Culturale "Pasquale Vignola", 1989. Os primeiros resultados do estudo foram apresentados em M. D'Alessio, Scuola e lingua nel Molise di fine Ottocento, Napoli, Edizioni Scientifiche Italiane, 2005, pp. 130-166. Cf. G. Palmieri, Berengario Galileo Amorosa. L'intellettuale e lo storico, in Id., Studi di storiografia molisana, Campobasso, Palladino Editore, 2016, pp. 89-105.

${ }^{14}$ Cf. A. Santoriello, Berengario Galileo Amorosa, in Id. (Ed.), Riccia tra biografie e storia. Ricerche su personaggi illustri dell'Ottocento e del Novecento, Riccia, Associazione culturale "Pasquale Vignola", 1997, pp.196-197 e Id., I fratelli Amorosa, Ibid., pp. 125-136.

${ }^{15}$ Sobre o exaltado ginásio-liceu da capital de Molise, remete-se à reconstrução apresentada no volume $M$. D'Alessio, Vita tra i banchi nell'Italia meridionale. Culture scolastiche in Molise fra Otto e Novecento, prefácio de Alberto Barausse, Coleção Biblioteca do "Centro di documentazione e ricerca sulla storia delle istituzioni scolastiche, del libro scolastico e della letteratura per l'infanzia" da Università degli Studi del Molise, Campobasso, Palladino Editore, 2011, pp. 13-44.

${ }^{16}$ De $1^{\circ}$ de novembro de 1884 a 31 de outubro de 1889 . Os documentos relacionados aos primeiros anos da prática de ensino de Amorosa estão preservados no arquivo histórico da comuna de Riccia: Berengario Galileo Amorosa, in Istruzione pubblica: maestri (1806-1896), b. 110, f. 761.
} 
(de 1898 até 1905$)^{17}$, durante o qual contribuiu para o Bollettino magistrale local com artigos "de índole didática e de política da educação”.

No prefácio de suas memórias, Amorosa fala da reduzida remuneração concedida a um inspetor em sua primeira tarefa, com salário de 1.500 liras e da mais árdua jornada, que parecia trazer pouco progresso à carreira, fortemente condicionados pelo pequeno número de funcionários previsto à época ${ }^{18}$, até a promoção ao papel de Superintendente após cerca de quinze anos de peregrinações de norte a sul da Itália, chegando a receber 2.000 liras.

Eloquente orador, historiador, acadêmico e escritor, Amorosa uniu a personalidade de intelectual propenso ao estudo e ao multifacetado compromisso cultural aos traços maiores de uma figura eminente do mundo escolar. Em 1903, foi aprovado no concurso realizado naquele ano para oito vagas de superintendência de educação, e depois de renunciar à posição em Caltanissetta, transferiu-se para Catanzaro de 1905 a 1908. Retornou a Abruzzo, permanecendo em Chieti por cinco anos, de 1908 a 1913, e dali passou a Caserta por três anos, até 1919. Foi então chamado para a direçãogeral das escolas italianas no exterior, mas renunciou por razões familiares ao trabalho nas escolas da América Latina (mantendo-se no Ministério até 1920). Recupera o posto de superintendente em Chieti, onde permaneceu até 30 de junho de 1923. Aposentou-se aos 58 anos de idade em dezembro de 1923, depois de ser chamado para fazer parte da Comissão Central para a revisão de livros didáticos, cuja primeira sessão foi presidida pelo próprio Giuseppe Lombardo Radice.

\footnotetext{
${ }^{17}$ Sobre esses anos, recomenda-se G. Palmieri, Il contributo della «Rivista abruzzese agli studi del Molise», in «Rivista storica del Sannio», a. VIII (2000), n. 13, pp. 305-323.

${ }^{18}$ O Decreto Real de 29 de setembro de 1885 previa um quadro de funcionários, que durou por dez anos, de 25 inspetores de primeira classe, 35 de segunda, 87 de terceira, 91 de quarta. A remuneração da classe mais elevada era de 3.000 liras. O Decreto Real de 8 de agosto de 1895 reduziu os inspetores a 218, formando três classes. Uma coleção útil das disposições sobre "serviço de inspeção" até os anos 1920 está em G Carenzi, Manuale di legislazione scolastica, Torino, Paravia, 1919 (o segundo volume é de 1920), especialmente o segundo capítulo. Para uma discussão completa sobre aspectos relativos ao "tema da função de supervisão escolar" de acordo com a legislação italiana e com referências à legislação de alguns países estrangeiros, indica-se duas obras específicas, muito bem documentadas e que se complementam: cf. A. Saraz, La tecnica dell'ispezione scolastica, Torino, G.B. Paravia \& C., 1921 e Id., Vicende storiche ed attuale ordinamento della Direzione ed Ispezione scolastica, Torino, G.B. Paravia \& C., 1922.
} 
A ampla produção de Amorosa abrange aquela inicial, de cunho poético ${ }^{19}$, aquela posterior, jornalística, histórica e erudita em prosa, interessada nas tradições folclóricas de Molise $^{20}$, chegando àquela destinada expressamente à escola, de acordo com uma grande variedade cultural e estilística.

\section{A produção de cunho escolar}

O impacto do componente escolar é atestado pela variedade e continuidade dos escritos dedicados por Amorosa à discussão desse tema. Estes incluem os primeiros artigos publicados em importantes revistas pedagógicas nacionais, entre as quais estão Avvenire educativo, Rinnovamento scolastico e Risveglio educativo. Abrangem ainda as histórias sobre o mundo escolar e seus principais jogadores, inspiradas nas várias experiências vividas nos muitos lugares com os quais o autor entrou em contato e coletadas sob a forma narrativa breve de perfil biográfico, ensaio e esboço no volume Paria moderno. Documenti umani ${ }^{21}$, em cujas páginas são relatadas histórias de vida reais de muitos professores e professoras "párias", vítimas de discriminação e injustiça social na Itália pós-unificação. Sob essa mesma chave narrativa e evocativa pessoal, como haverá melhor maneira de ilustrar, as memórias de II duro calle. Memorie d'un ispettore scolastico, publicadas em 1912, propõem o autor como narrador externo ao mesmo tempo que personagem interno ao mundo que narra. As intervenções dedicadas especificamente às questões educacionais e pedagógicas debatidas nos primeiros vinte anos do século XX são coletadas nas Note didattiche e di politica scolastica, publicadas em $1903^{22}$, e no volume posterior à Primeira Guerra Mundial, La scuola in provincia di Terra di Lavoro nei primi tre anni di guerra, de $1918^{23}$.

Além dessas obras, os dois principais volumes de destinação educacional específica de Molise e Abruzzo ocupam um lugar próprio - dado o tipo original de texto e

\footnotetext{
${ }^{19}$ B.G. Amorosa, Epodon: versi, Conegliano, Tip. F. Cagnani, 1891; Id., Cadore. Impressioni liriche, Pieve di Cadore, E. Berengan e C., 1893.

${ }^{20}$ Id., Riccia nella storia e nel folklore, Casalbordino, Tip. N. De Arcangelis, 1903.

${ }^{21}$ Palermo, Sandron, 1895.

${ }^{22}$ Saggi critici, Lanciano, Tip. A. Rosati, 1903.

${ }^{23}$ Note ed appunti del R. Provveditore agli Studi Dott. B.G. Amorosa, Caserta, Tipografia Economica B. De Simone, 1918.
} 
o conteúdo principal de cultura regional -, que foram publicados por Amorosa entre 1924 e 1925 na prestigiada coleção Mondadori de almanaques regionais ${ }^{24}$. No contexto da produção editorial renovada voltada ao estudo da cultura regional segundo o ditado pelos programas lombardos, ambas as cartilhas conotavam sentido marcadamente antropogeográfico, deixando amplo espaço para as práticas locais, costumes, poesia dialetal, rituais tradicionais, além do acúmulo de materiais eruditos caros ao autor. Retorna nessas páginas aquele tipo de compromisso descoberto na prática escrita de Amorosa, entre a tendência à invenção e a pesquisa histórico-erudita, uma tensão contraposta entre a disponibilidade para a obra por si literária e a inclinação à observação direta da realidade social, educacional ou folclórica ${ }^{25}$.

\section{Obras de inspeção: uma frente investigativa negligenciada}

Tentando retomar na parábola pessoal do funcionário italiano a frente investigativa, que se destaca por ser considerada apenas marginalmente pela historiografia do setor, será útil classificar os diversos tipos de texto que podem ser isolados no rol geral de obras de inspeção. É importante reafirmar como o traço preeminente que aqui se pretende enfatizar com os movimentos da experiência de memórias autobiográficas do molisano é o plano das representações de si em relação ao mundo escolar, assim como emerge de diversos lugares textuais. Atendo-se, portanto, aos trabalhos criados pela pena de inspetores que operam em diversas áreas da "Italia delle Italie", tentar-se-á esclarecer os principais.

\footnotetext{
24 B.G. Amorosa, II Molise. Almanacco regionale, Mondadori, 1924, posteriormente reimpresso com introdução de Giulio Di Iorio, da Associazione Culturale "P. Vignola", Riccia, em 1990. Di lorio examina como "os maiores incentivos para escrever um livro de cultura regional vêm daquelas intenções didáticas internas ao Amorosa - por profissão no ápice do sistema educacional - sempre atento seja à questão da escola, seja àqueles que nela trabalham todos os dias. $\mathrm{E}$ aos professores Amorosa dedicou uma coleção de histórias curtas em que ele descreveu, embora com muita ênfase, o seu difícil e duro trabalho quase sempre invisível para os outros" (pp. XIX-XX). No ano seguinte, Amorosa viu aprovado também o almanaque para a região de Abruzzo. Referências aos dois almanaques aprovados por Amorosa, comparados especialmente com o outro de Eugenio Cirese sempre em relação a Molise, estão contidas em A. Barausse, M. D'Alessio, "Dalla piccola alla grande patria". Libri dialettali e almanacchi regionali per la scuola elementare, in G. Chiosso (Ed.), TESEO '900. Editori scolastico-educativi del primo Novecento, Milano, Editrice Bibliografica, 2008, especialmente à p. XLIII.

${ }^{25}$ Considerações aprofundadas podem ser encontradas em M. D’Alessio, A scuola fra casa e patria, cit., pp. $133-137$.
} 
No primeiro corpus textual estão as minutas e os relatórios das visitas de inspeção que oferecem uma observação estruturada do progresso das escolas em determinado lugar e em determinado tempo. Considera-se nesse círculo a importância das atividades de supervisão e controle realizadas pela equipe da Associazione Nazionale per gli Interessi del Mezzogiorno d'Italia nas regiões em que durante o fascismo assegura a delegação para combater o analfabetismo ${ }^{26}$.

Logo depois estão os diários, as autobiografias, as memórias. Ao lado do "árduo caminho" de Amorosa não se pode negligenciar uma série de outros escritos de funcionários centrais e periféricos que conheceram as honras da imprensa. É o caso, por exemplo, do inspetor lucano Antonio Renzi que, com ainda outras obras sobre a história, a geografia e a cultura regional, entregou no final da sua carreira um interessante livro de memórias sobre aquilo a que ele chama, desde o título, sua obra de "apostolado" 27 , muito semelhante em tom e nos eventos narrados à exposição do inspetor de Molise. $\mathrm{O}$ mesmo Renzi, evidentemente bem estabelecido nos ambientes institucionais e editoriais da época, é autor recorrente da sessão “Inspecionando" da revista Tecnica Scolastica ${ }^{28}$, recaindo no terceiro espaço textual identificado, aquele dos artigos e ensaios de carácter técnico.

Aqui estão também as várias monografias acolhidas em uma coleção específica de textos de inspetores e vice-inspetores de tema pedagógico e didático-educativo. A “Biblioteca degli ispettori scolastici" foi publicada em 1912 pelo editor Antonio Vallardi que, na apresentação, especificava como o projeto compreendia "os escritos dos inspetores e vice-inspetores, sem precondição pedagógica ou educacional ou filosófica ou científica ou social. Acolhe, portanto, com completa objetividade e imparcialidade, as manifestações das mais diferentes escolas ou teorias ou tendências, fornecendo como único limite a unidade orgânica do trabalho que propõem os distintos autores". Os trabalhos encontrados cobrem um período de mais de dez anos (de 1912 a 1923),

\footnotetext{
${ }^{26}$ Cf. M. D'Alessio, Our Schools. The work of the Association of Southern Italy against illiteracy in Basilicata (1921-1928), in «History of Education \& Children's Literature», a. X, n. 2 (2015), pp. 463-480 ed Ead., Igiene e scuole rurali. Itinerari ed esperienze dell'ANIMI in Basilicata durante il fascismo tra educazione e propaganda, in «Rivista di Storia dell’Educazione», n. 2 (2016), no prelo.

27 A. Renzi, “Apostolato". Memorie autobiografiche di Antonio Renzi, Regio Ispettore scolastico a riposo, Minerbio, Tip. Bevilacqua, [1930].

${ }^{28}$ A. Barausse, M. D’Alessio, "La salvezza della scuola verrà dai tecnici”. La stampa pedagogica in Italia tra idealismo e fascismo: la «Tecnica scolastica (1923-1927), cit.
} 
atentando-se ao principal período de transição do idealismo ao fascismo, e documentam um interesse contínuo e sistemático para o exercício da inspetoria técnica e educacional, desenvolvido em dezesseis volumes publicados pelos tipos vallardianos ${ }^{29}$. O quarto tipo identificado é aquele, já aludido nas páginas de Paria moderno de Amorosa, dos contos, das novelas, dos romances e dos livros didáticos ${ }^{30}$.

Em todos os textos, exemplos dos diversos planos de trabalho profissional e literário de inspetores italianos, é importante destacar a mobilidade do ponto de vista dos autores. Parte-se daquilo que podemos definir como observação objetiva, funcional para a tarefa de "informar" pontualmente no desenvolvimento da atividade de supervisão e controle; a "subjetividade" divergente encontrada na exigência de "contar" as experiências de vida, em particular desenvolvidas enquanto se mostra os arredores à classe dos professores; o papel de "mediação" desempenhado em fases que visam “educar", exercendo a função técnica específica, tendo em conta a formação da consciência profissional; e, finalmente, a veia narrativa específica, detectável na reelaboração literária das crônicas, também visando educar todos os operadores da vida escolar sobre a consciência educacional pública.

Portanto, é possível acompanhar a expansão do olhar dos funcionários ministeriais que se origina do próprio narrador, passa à consciência de pertencer à categoria profissional, ao mundo das escolas e dos professores, até chegar à sociedade e, a partir dali, à história.

\footnotetext{
${ }^{29}$ A série começou com algumas "recomendações e normas didáticas" voltadas para jovens professores novatos e continuou, além de trabalhos marcados por essa linha de sugestões práticas dadas pelos inspetores, com volumes de "provocações e ideias educacionais" e páginas mais propriamente pedagógicas até os escritos educativos e "de arte diadática". Cf. D. Borghese, Lettere a una giovane normalista: (dalle rive della Dora), Milano, A. Vallardi, 1912, Biblioteca degli ispettori scolastici 1; G. Antonietti, Dal taccuino di un ispettore: noterelle didattiche, Ibid., 1913, 2; F. Esposito, La suggestione come mezzo educativo, Ibid., 1914, 3; V. Norscia, Nel campo della pedagogia, Ibid., 1914, 4; G. Simeoni, Consigli e norme didattiche agli insegnanti esordienti, Ibid., 1915, 5; A. Piccioni, Punzecchiature e spunti scolastici, Ibid., 1915, 6; P. Carlini, Del carattere come formazione psicologica: con l'appendice di una conferenza didattica e due discorsi, Ibid., 1915, 7; D. De Murtas, L'emozione estetica considerata come mezzo di educazione: note di fisio-psicologia, Ibid., 1915, 8; P. Cavazzuti, Il patronato scolastico e le più importanti istituzioni ausiliarie della scuola in Italia, Ibid., 1916, 9; I. Dossi, Pagine di pedagogia di un educatore trentino, Ibid., 1919, 10; F. Bianchi, Aristide Gabelli nella filosofia positiva e nella pedagogia applicata, Ibid., 1920, 12; R. Mariani, Lettere didattiche ad un'esordiente di scuola rurale, Ibid., 1920, 13; A. Bianchessi, Scritti educativi, ivi, 1920, 14; A.F. Cossu, Note e riflessi di arte didattica, Ibid., 1923, 16.

${ }^{30}$ Sobre a redescoberta da obra escolar mencionada acima, cf. M. D'Alessio, Scuola e lingua nel Molise di fine Ottocento, cit., especialmente pp. 142-166.
} 


\section{5. "O árduo caminho" dos inspetores: o compromisso "para aumentar o amor pela escola, pelos professores e pelos inspetores"}

As histórias do inspetor molisano são organizadas em um grande volume de cerca de 300 páginas distribuídas em 31 capítulos, que se estendem das memórias iniciais da sua própria família às diversas etapas de sua formação, até a recordação das primeiras tarefas de inspeção desde o Veneto até Abruzzo.

O ritmo da história é confiado a uma chave evocativa e narrativa pessoal que não podem naturalmente prescindir do âmbito da experiência coletiva que ocorre em segundo plano, aproveitando contextos locais individuais no amplo espectro da escola nacional. Neste sentido, adquire importância o trabalho de Amorosa, que reflete fielmente

catorze anos de vida dedicada ao ensino fundamental, entre povos e cidades distintos, viajando no inverno e no verão, enfrentando sacrifícios e privações, muitas vezes lutando contra a fúria dos elementos e constantemente contra a apatia ou aversão dos homens ${ }^{31}$.

O livro fornece uma representação precisa do "ambiente complexo" com que o inspetor entrou em contato ao longo de suas viagens aos diversos locais de trabalho. Assim, à reconstrução da trajetória existencial pessoal e profissional se associa a descrição de uma notável capacidade de estabelecer relacionamentos frutíferos e interagir "intensamente com todos os lugares em que se vive e trabalha procurando, ao mesmo tempo, compreender as características que sugerem a história e a cultura desses lugares e confronta-los, 'dialogar' com eles por meio do estudo e da escrita”32.

A fisionomia da missão da inspeção é bem definida pelo autor nos anos em que "ninguém pensava na escola e nos mestres e não haviam surgido ainda as poderosas associações que [...] sabiam como sacudir a indiferença dos governantes e estimular algumas leis providentes"33:

\footnotetext{
${ }^{31}$ B.G. Amorosa, "Il duro calle", cit., p. VI.

${ }^{32}$ G. Palmieri, Berengario Galileo Amorosa. L'intellettuale e lo storico, cit., p. 91.

${ }^{33}$ B.G. Amorosa, "Il duro calle", cit.
} 
o legislador tinha atribuído a ele uma missão técnica, na acepção mais nobre e mais verdadeira da palavra. Teria que carregar entre os bancos da escola o contributo da sua competência profissional, apoiar e corrigir os professores na implementação de bons métodos, orientar a arte didática no que concernia às novas teorias da ciência da educação, difundir e ilustrar entre seus funcionários o pensamento dos pedagogos e a mudança e crescimento progressivos dos programas, popularizar entre as massas o carinho pela escola e por ela arrancar das autoridades municipais todos os meios possíveis ${ }^{34}$.

Mas a respeito desses demais objetivos, o peso de toda a burocracia, feita de tarefas, papeis e estatísticas sem fim, como o próprio Amorosa expõe, tinha subtraído “no ciclone de papel e tinta" - muito da eficácia da obra técnica e educacional do inspetor, que ao longo do tempo se reduzira principalmente a "relatar"35.

A meio caminho entre registro e memória pessoal, o conhecimento sobre o mundo escolar é restituído pelos traços realistas de rostos e acontecimentos e das histórias de um observador "especial": emerge uma representação da escola entre o fim do século XIX e início do século XX que transmite informações sobre o cotidiano escolar sobretudo nos lugares distantes dos principais centros da vida política, administrativa e educacional.

Fatos e situações concretos são relatados em termos de aplicações práticas das orientações didático-pedagógicas: consulte o capítulo sobre "I broccoli e le acacie di S.E. Baccelli", com o "novo verbo" com o qual em 1898 se fez "as novas gerações se apaixonarem pelos campos".

O inspetor então relata notícias sobre o desempenho das escolas, informando-nos, por exemplo, que uma das escolas rurais visitadas se situava em um "estábulo de seis ou sete metros quadrados, com uma manjedoura onde duas ovelhas pastam a grama fresca: nove ou dez crianças estavam agachadas no chão. O professor [...] gritava como um leiloeiro, lendo uma página do silabário que as crianças repetiam em coro" ${ }^{36}$. Atendo-se

\footnotetext{
${ }^{34}$ Ibid., p. VII.

35 B.G. Amorosa, "Il duro calle", cit., p. IX. Amorosa recorda com certa amargura as transformações que levaram o inspetor a "se tornar um escrivinhador" e como nem mesmo a lei de 24 de dezembro de 1904 Ihes havia restituído a sua aparência original. As condições financeiras e de carreira, aliás, não foram melhoradas, uma vez que, suprimida a classe de L. 2000, formaram-se duas de 113 lugares cada, até a promulgação da lei sobre o estado econômico em 30 de junho de 1908 n. 304 . Ibidem.

${ }^{36}$ Ibid., p. 202.
} 
às condições dos locais e dos móveis descreve a escola que "foi colocada em uma casa de campo sem forma [...] ratos e aranhas estavam confortavelmente instalados e o sabiam os pobres cadernos roídos e alguns cartazes do alfabeto muito antigos cobertos de teias de aranha" 37 . Sobre as práticas didáticas o capítulo "Metodo fonico" parece significativo, em que se denuncia a "duplicação e fortalecimento [...] de distinção acadêmica de nenhum valor".

Mas outras inserções da vida escolar apresentam os casos dos professores, com suas doenças ou calúnias de que eram objeto, a dificuldade na escolha dos livros didáticos, certas figuras entre os alunos, bem como a avaliação das condições dos professores, lutando pelo reconhecimento de sua profissão do ponto de vista econômicojurídico e social nas pequenas comunidades onde eram chamados para trabalhar e viver.

Provenientes de observadores e narradores dos principais processos de renovação educacional italiana entre os séculos XIX e XX, as memórias de inspeção escolar - exemplificadas pelo volume do autor de Molise - sugerem um gênero textual de literatura escolar menor certamente merecedor de mais atenção e que não deve, absolutamente, ser negligenciado. As atitudes literárias e educacionais encontradas nessas páginas exibem o domínio de uma prosa controlada e de um código de altamente expressivo, às vezes retórico, sempre fortemente literário e "carducciano" que é acompanhado por uma disposição narrativa recorrente que tende à "medida breve" que pode ser estendida, de forma especular, à pena dos inspetores italianos. O livro de memórias de inspeção, pretende-se destacar, baseia-se nos modelos literários do final do século XIX e combina o estilo em prosa com a necessidade de uma representação mais ampla e realista dos momentos que marcaram a experiência individual em eventos coletivos da escola nacional, assim como é evocado pela história. Ao longo dos capítulos da difícil peregrinação “pelas escadas alheias" se enrola junto à duradoura fidelidade a um modelo de literatura e escrita transmitida por uma forte erudição retórico-humanista um claro compromisso do corpo diretivo e de gestão, de cunho profissional, e de altos “ideais educacionais".

\footnotetext{
37 Ibid., p. 132.
} 


\section{Conclusões}

Parece evidente que o dualismo da memória escolar observado até agora que emerge das páginas de Amorosa não marca uma distância entre o autor e o ambiente representado, mas, em vez disso, permite uma leitura atenta e detalhada das condições da escola real, em relação àquela legal entregue pelas disposições normativas e pelos programas, há muito considerada homogênea em todo o país. Sobretudo, da história e dos eventos narrados emerge claramente o papel educativo mais amplo dos inspetores escolares que, em seu longo "aprendizado científico e profissional", se mostram os maiores conhecedores do mundo escolar e reais autores da obra de unificação cultural e pedagógica do país.

Nesse sentido, é possível concluir que por meio de obras memoriais como aquela de Amorosa se pode rastrear a passagem da memória para a história. O "árduo caminho" se origina em uma forma inicial de memória individual do inspetor italiano, zelosa para retribuir, em particular, toda a dívida de reconhecimento para com a figura do pai, mestre, que deixou de herança um "legado de integridade e dever”; se move em direção à esfera de memória corporativa profissional, no compromisso com a formação da consciência da categoria dos inspetores ${ }^{38}$; avança até a dimensão da memória coletiva de uma representação de um esboço temporal e social da escola italiana dos séculos XIX e XX. À memória pessoal se somam as memórias múltiplas da história em comum, na ótica das transformações historiográficas que têm recentemente enfatizado o papel da educação e da história da escola em relação à vida expandida da sociedade.

As memórias do inspetor molisano, finalmente, contribuíram enquanto gênero textual que pode abranger ampla gama de escritos afins encontrados na literatura escolar, muitas vezes deixada à sombra de funcionários e executivos nacionais e periféricos, para avaliar os inspetores através de quais formas e experiências, no exercício

\footnotetext{
${ }^{38}$ Recorde-se que na primavera de 1902 foi instituída em Milão a Associazione Nazionale fra gli Ispettori Scolastici (ANIS), sob a direção do inspetor central Comm. Graziani, vice-presidente, e de Tullio Fontana, secretário. Para notícias sobre a necessidade dos inspetores "de se organizar para proteger seus próprios interesses morais e materiais, especialmente depois de ter assistido e contribuído para o despertar da classe magistral unida em poderoso organismo apto a chamar atenção da opinião pública e dos governantes sobre os problemas da escola", recomenda-se A. Saraz, Vicende storiche ed attuale ordinamento della Direzione ed Ispezione scolastica, cit., p. 127.
} 
técnico de uma função altamente social, ativamente contribuíram para a construção da visão nacional da escola e o professor.

Nesse sentido, o estudo de caso proposto nos entrega uma peça significativa de uma função, a da inspetoria, que particularmente na exemplificação de sua "técnica" se apresenta como uma "soma de conhecimentos, resultado de experiências, observações, estudos, meditações", e uma série de competências "adquiridas pelo exercício contínuo e uso de dons e aptidões exclusivamente pessoais"39.

Essa identidade da visão coletiva nacional do corpo profissional de inspeção comprova, em resumo, o extraordinário valor histórico e educacional dessa fonte, ao iluminar os acontecimentos pessoais e institucionais de protagonistas incontestáveis de dois séculos de vida educacional na Itália.

\section{Referências}

AMOROSA, Berengario Galileo. Autobiografia. In memoria del G. Uff. Dottor Berengario Galileo Amorosa Provveditore agli studi, MDCCCLIV-MCMXXXVII, s.n.t. [1938], pp. 11-23

AMOROSA, Berengario Galileo. II Molise. Almanacco regionale, Mondadori, 1924, poi ristampato con saggio introduttivo di Giulio DI IORIO, Riccia, Associazione Culturale " $P$. Vignola", 1990

AMOROSA, Berengario Galileo. La scuola in provincia di Terra di Lavoro nei primi tre anni di guerra. Note ed appunti del R. Provveditore agli Studi Dott. B.G. Amorosa, Caserta, Tipografia Economica B. De Simone, 1918

AMOROSA, Berengario Galileo. II “duro calle”. Memorie d'un ispettore scolastico, Milano Roma - Napoli, Società editrice Dante Alighieri di Albrighi \& C., 1912

AMOROSA, Berengario Galileo. Note didattiche e di politica scolastica. Saggi critici, Lanciano, Tip. A. Rosati, 1903

\footnotetext{
${ }^{39}$ A. Saraz, La tecnica dell'ispezione scolastica, cit., p. 1.
} 
AMOROSA, Berengario Galileo. Riccia nella storia e nel folklore, Casalbordino, Tip. N. De Arcangelis, 1903

AMOROSA, Berengario Galileo. Paria moderno. Documenti umani, Palermo, Sandron, 1895

AMOROSA, Berengario Galileo. Cadore. Impressioni liriche, Pieve di Cadore, E. Berengan e C., 1893

AMOROSA, Berengario Galileo. Epodon: versi, Conegliano, Tip. F. Cagnani, 1891

ANTONIETTI, Gaspare. Dal taccuino di un ispettore: noterelle didattiche, Milano, A. Vallardi, 1913, Biblioteca degli ispettori scolastici 2

BARAUSSE, Alberto. Nonostante tanto diluvio di libri scolastici. I libri di testo per le scuole elementari e le indagini ministeriali di Bargoni e Bonghi durante gli anni della Destra storica (1869-1875), Lecce, Pensa Multimedia, 2015

BARAUSSE, Alberto. I maestri all'università: la Scuola pedagogica di Roma 1904-1923, Perugia, Morlacchi, 2004

BARAUSSE, Alberto, D’ALESSIO Michela. “La salvezza della scuola verrà dai tecnici”. La stampa pedagogica in Italia tra idealismo e fascismo: la «Tecnica scolastica» (1923-1927). In HERNANDEZ DIAZ Jose Maria (coord.), La prensa de los escolares y estudiantes. Su contribucion al patrimonio historico educativo, Salamanca, Ediciones Universidad de Salamanca (Collecion Aquilafuente, 210), 2015, pp. 611-626

BARAUSSE, Alberto, D’ALESSIO, Michela. "Dalla piccola alla grande patria”. Libri dialettali e almanacchi regionali per la scuola elementare. In CHIOSSO Giorgio (coord.), TESEO ‘900. Editori scolastico-educativi del primo Novecento, Milano, Editrice Bibliografica, 2008

BANCHESSI, Antonio. Scritti educativi, Milano, A. Vallardi,1920, Biblioteca degli ispettori scolastici 14

BIANCHI, Francesco. Aristide Gabelli nella filosofia positiva e nella pedagogia applicata, Milano, A. Vallardi,1920, Biblioteca degli ispettori scolastici 12 BORGHESE, Domenico. Lettere a una giovine normalista: (dalle rive della Dora), Milano, A. Vallardi, 1912, Biblioteca degli ispettori scolastici 1

CARLINI, Pietro. Del carattere come formazione psicologica: con l'appendice di una conferenza didattica e due discorsi, Milano, A. Vallardi, 1915, Biblioteca degli ispettori scolastici 7

CAVAZZUTI, Pietro. Il patronato scolastico e le più importanti istituzioni ausiliarie della scuola in Italia, Milano, A. Vallardi,1916, Biblioteca degli ispettori scolastici 9 
CHIOSSO, Giorgio. Alfabeti d'Italia. La lotta contro l'ignoranza nell'Italia unita, Torino, SEI, 2011

CIVES, Giacomo. Cento anni di vita scolastica in Italia. Ispezioni e inchieste dall'idealisno a oggi negli scritti di G. Lombardo Radice, G. Isnardi, G. Giovinazzi, F. Betttini, A. Marcucci, L. Volpicelli, L. Borghi, A. Visalberghi, Roma, Armando Editore, 1967

CIVES, Giacomo. Cento anni di vita scolastica in Italia. Ispezioni e inchieste da Gino Capponi a Giuseppe Lombardo Radice, Roma, Armando Editore, 1960

COSSU, Anton Francesco. Note e riflessi di arte didattica, Milano, A. Vallardi,1923, Biblioteca degli ispettori scolastici 16

D'ALESSIO, Michela. Igiene e scuole rurali. Itinerari ed esperienze dell'ANIMI in Basilicata durante il fascismo tra educazione e propaganda. In Atti del Convegno CIRSE Centro Italiano per la Riceraca Storico Educativa, Sguardi della storia. Luoghi, figure, immaginario e teorie dell'educazione (Bologna, 26-27 febbraio 2016). «Rivista di Storia dell'Educazione», v. 3, n. 2 (2016), pp. 43-48

D'ALESSIO, Michela. Our Schools". The work of the Association of Southern Italy against illiteracy in Basilicata (1921-1928). «History of Education \& Children's Literature», a. X, n. 2 (2015), pp. 463-480

D’ALESSIO, Michela. “Il duro calle”. Memorie di un ispettore scolastico (Berengario Galileo Amorosa, 1865-1937). In YANES, Cristina, MEDA, Juri (coords.), International Symposium, School memories. New trends in Historical research into Education Heuristic Perspectives and Methodological issues, (Siviglia, 22-23 settembre 2015). Abstracts, p. 22

D'ALESSIO, Michela. Life at school: class registers as a new source of studying historical and educational heritage. In BADANELLI RUBIO, Ana M., POVEDA SANZ Maria, RODRIGUEZ GUERRERO Carmen (coords.), Pedagogia museistica. Practicas, usos didacticos e investigacion del patrimonio educativo. Atti della VI Jornadas cientifica della Sociedad Espanola para el Estudio del Patrimonio Historico educativo SEPHE, Madrid, 2224 ottobre 214, Madrid, Universidad Complutense de Madrid, Facultad de Educacion, 2014, pp. 401-409

D'ALESSIO, Michela. A scuola fra casa e patria. Dialetto e cultura regionale nei libri di testo durante il fascismo, Collana Biblioteca del "Centro di documentazione e ricerca sulla storia delle istituzioni scolastiche, del libro scolastico e della letteratura per l'infanzia" dell'Università degli Studi del Molise, Lecce, Pensa Multimedia, 2013

D'ALESSIO, Michela. Berengario Galileo Amorosa, in CHIOSSO, Giorgio e SANI, Roberto (coords.). DBE - Dizionario Biografico dell'Educazione. 1800-2000, Milano, Editrice Bibliografica, 2013, 2 voll., v. I, scheda n. 75, pp. 45-46 
D'ALESSIO, Michela. Vita tra i banchi nell'Italia meridionale. Culture scolastiche in Molise fra Otto e.Novecento, premessa di Alberto BARAUSSE, Collana Biblioteca del "Centro di documentazione e ricerca sulla storia delle istituzioni scolastiche, del libro scolastico e della letteratura per l'infanzia" dell'Università degli Studi del Molise, Campobasso, Palladino Editore, 2011, pp. 13-44

D’ALESSIO, Michela. Scuola e lingua nel Molise di fine Ottocento, Napoli, Edizioni Scientifiche Italiane, 2005

DECOLLANZ, Giuseppe. La funzione ispettiva dalla legge Casati ad oggi, Roma, Armando, 1984

DE MURTAS, Daniele. L'emozione estetica considerata come mezzo di educazione: note di fisio-psicologia, Milano, A. Vallardi, 1915, Biblioteca degli ispettori scolastici 8

DOSSI, Ilario, Pagine di pedagogia di un educatore trentino, Milano, A. Vallardi, 1919, Biblioteca degli ispettori scolastici 10

ESPOSITO, Francesco. La suggestione come mezzo educativo, Milano, A. Vallardi,1914, Biblioteca degli ispettori scolastici 3

MARIANI, Raffaele. Lettere didattiche ad un'esordiente di scuola rurale, Milano, A. Vallardi,1920, Biblioteca degli ispettori scolastici 13

NORSCIA, Vincenzina. Nel campo della pedagogia, Milano, A. Vallardi,1914, Biblioteca degli ispettori scolastici 4

LOMBARDO RADICE, Giuseppe. Presentazione a DAL PIAZ, Riccardo. Esperienze didattiche di un ispettore trentino. Relazione-studio sulle scuole della circoscrizione di Trento (1925-1926), Roma, Associazione per il Mezzogiorno, 1928, p. 3

PALMIERI, Giorgio. Berengario Galileo Amorosa. L'intellettuale e lo storico. In Id., Studi di storiografia molisana, Campobasso, Palladino Editore, 2016, pp. 89-105

PALMIERI, Giorgio. II contributo della «Rivista abruzzese agli studi del Molise». «Rivista storica del Sannio», a. VIII (2000), n. 13, pp. 305-323

PALMIERI, Giorgio, SANTORIELLO, Antonio (coords.). Berengario Galileo Amorosa. Atti del Convegno (Riccia, 18 luglio 1987), Riccia, Associazione Culturale "Pasquale Vignola", 1989

PICCIONI, Augusto. Punzecchiature e spunti scolastici, Milano, A. Vallardi,1915, Biblioteca degli ispettori scolastici 6

RENZI, Antonio. “Apostolato". Memorie autobiografiche di Antonio Renzi, Regio Ispettore scolastico a riposo, Minerbio, Tip. Bevilacqua, [1930] 
SANTORIELLO, Antonio. Berengario Galileo Amorosa. In Id. (coord.), Riccia tra biografie e storia. Ricerche su personaggi illustri dell'Ottocento e del Novecento, Riccia, Associazione culturale "Pasquale Vignola”, 1997, pp.196-197

SANTORIELLO, Antonio. I fratelli Amorosa. In Id. (coord.), Riccia tra biografie e storia. Ricerche su personaggi illustri dell'Ottocento e del Novecento, Riccia, Associazione culturale “Pasquale Vignola”, 1997, pp. 125-136

SARAZ, Alfredo. Vicende storiche ed attuale ordinamento della Direzione ed Ispezione scolastica, Torino, G.B. Paravia \& C., 1922

SARAZ, Alfredo. La tecnica dell'ispezione scolastica, Torino, G.B. Paravia \& C., 1921

SIMEONI G[s.n.]. Consigli e norme didattiche agli insegnanti esordienti, Milano, A. Vallardi, 1915, Biblioteca degli ispettori scolastici 5

VIGNAO FRAGO, Antonio. Relatos y relaciones autobiograficas de profesores y maestros. In ESCOLANO BENITO, Augustin, HERNANDEZ DIAZ, Jose Maria (coords.), La memoria y el deseo. Cultura de la escuela y educacion deseada, Valencia, Tirant Lo Blanch, 2002, pp. $135-175$. 